PUPT-1933

\title{
Loop dynamics and AdS/CFT correspondence
}

\author{
A. M. Polyakov ${ }^{1}$ and V. S. Rychkov ${ }^{2}$ \\ 1 Joseph Henry Laboratories, Princeton University, Princeton, NJ 08544 \\ polyakov@viper.princeton.edu \\ ${ }^{2}$ Department of Mathematics, Princeton University, Princeton, NJ 08544 \\ rytchkov@math.princeton.edu
}

\begin{abstract}
We consider the strong coupling limit of conformal gauge theories in 4 dimensions. The action of the loop operator on the minimal area in the AdS space is analyzed, and the Schwinger-Dyson equations of gauge theory are checked. The general approach to the loop dynamics developed here goes beyond the special case of conformal theories.
\end{abstract}

May 2000 


\section{Introduction}

In spite of the great recent progress in understanding gauge fields-strings connection and its special case-AdS/CFT correspondence, there is still no true derivation of these phenomena based on the first principles. In the paper [四] we made a step in this direction by demonstrating that the loop equations of gauge theory can be verified on the string side in WKB approximation and for the special contours (wavy lines).

In this note we will give a general treatment of the problem valid for any loops. Our new approach turns out to be simpler. It reveals some amazing features, connecting the loop Laplacian and the minimal area functional in the AdS space. As a result, we will be able to check the loop equations in WKB approximation for arbitrary non-selfintersecting loops. There is a direct path from here to the full quantum theory, but it will be left for the future, apart from several comments.

Let us first formulate our main results. Consider the Wilson loop

$$
W[C]=\frac{1}{N}\left\langle\operatorname{Tr} P \exp \oint_{C} A_{\mu} d x^{\mu}\right\rangle,
$$

where we average the ordered exponential over the Yang-Mills fields. In this pure gauge theory (1.1) satisfies the loop equation

$$
\widehat{L}(s) W[C]=W * W
$$

where the RHS is zero for non-selfintersecting loops and the loop Laplacian $\widehat{L}$ is defined by

$$
\frac{\delta^{2} W}{\delta c_{\mu}(s) \delta c_{\mu}\left(s^{\prime}\right)}=(\widehat{L}(s) W) \cdot \delta\left(s-s^{\prime}\right)+\text { non-local terms } .
$$

The decomposition (1.3) is not always possible - its existence is characteristic of the zigzaginvariant functionals [1].

When there are other fields in the theory (like in $\mathcal{N}=4 \mathrm{SYM}$ ) one can define many different loop functionals, like the one introduced in [2], [3]

$$
\widetilde{W}[c(s), y(s)]=\frac{1}{N}\left\langle\operatorname{Tr} P \exp \oint_{C}\left(A_{\mu} d x_{\mu}+\Phi_{a} d y_{a}\right)\right\rangle .
$$

In principle we can integrate around the loop any operator in the adjoint representation and thus define infinitely many various "Wilson loops". It is an open question which one of them 
should be used in the gauge fields - strings correspondence. This question is important for the full treatment of the problem. However, we conjecture that in the WKB approximation the asymptotic behavior for all reasonable definitions should be

$$
W[C] \propto e^{-\sqrt{\lambda} A_{\min }[C]},
$$

and in the same approximation

$$
\widehat{L}(s) W[C] \approx 0
$$

We are assuming here that we are dealing with any conformal version of gauge theory. The coupling is strong and $\lambda \gg 1$. When the couplings are running, the WKB approximation in general is not applicable.

In the second variational derivative of $W$ :

$$
\frac{\delta^{2} W}{\delta c_{\mu}(s) \delta c_{\mu}\left(s^{\prime}\right)}=\left(\lambda \frac{\delta A}{\delta c_{\mu}(s)} \frac{\delta A}{\delta c_{\mu}\left(s^{\prime}\right)}-\sqrt{\lambda} \frac{\delta^{2} A}{\delta c_{\mu}(s) \delta c_{\mu}\left(s^{\prime}\right)}\right) W
$$

the first term in brackets has no singularity for $s \rightarrow s^{\prime}$ and does not contribute to $\widehat{L}(s)$. Thus, the check that string theory in AdS space satisfies the loop equations of motion of a gauge theory reduces to the problem of calculating

$$
\widehat{L}(s) A_{\min }[c(s)]=?
$$

Solving this problem is the main objective of the present work.

\section{String Lagrangians}

Let us begin with setting up the general framework (see also [1]). The minimal area can be described with the use of the Dirichlet functional

$$
S=\frac{1}{2} \int_{\mathcal{D}} d^{2} \xi G_{M N}(x(\xi)) \partial_{a} x^{M}(\xi) \partial_{a} x^{N}(\xi)=\frac{1}{2} \int_{\mathcal{D}} \frac{d^{2} \xi}{y^{2}}\left[\left(\partial_{a} \boldsymbol{x}\right)^{2}+\left(\partial_{a} y\right)^{2}\right],
$$

\footnotetext{
${ }^{1}$ This fact was considered in $[6]$ as a check that the loop equation is satisfied. Notice however that in this order an arbitrary functional $A$ will pass this check.
} 
where $x^{M}=(y, \boldsymbol{x})=\left(y, x^{m}\right)$ are the coordinates in the $(D+1)$-dimensional AdS space with the metric $G_{M N}=y^{-2} \delta_{M N}(M=0, \ldots, D, m=1, \ldots, D)$. This functional must be minimized with the boundary conditions

$$
\left.\boldsymbol{x}\right|_{\partial \mathcal{D}}=\boldsymbol{c}(\alpha(s)),\left.\quad y\right|_{\partial \mathcal{D}}=0,
$$

where the reparametrization $\alpha(s)$ must be chosen so that the classical action

$$
F[\boldsymbol{c}(\alpha(s))]=\min _{\{\boldsymbol{x}, y\}} S[\boldsymbol{x}(\xi), y(\xi)]
$$

is stationary. That gives the minimal area

$$
A[\boldsymbol{c}(s)]=\min _{\{\alpha(s)\}} F[\boldsymbol{c}(\alpha(s))]
$$

Minimization (2.4) is equivalent to the Virasoro constraints imposed on the classical solution

$$
\left\{\begin{array}{l}
T_{\perp \perp}=\frac{1}{y^{2}}\left[\left(\partial_{\tau} \boldsymbol{x}\right)^{2}+\left(\partial_{\tau} y\right)^{2}-\left(\partial_{\sigma} \boldsymbol{x}\right)^{2}-\left(\partial_{\sigma} y\right)^{2}\right]=0 \\
T_{\perp \|}=\frac{1}{y^{2}}\left[\partial_{\tau} \boldsymbol{x} \partial_{\sigma} \boldsymbol{x}+\partial_{\tau} y \partial_{\sigma} y\right]=0
\end{array}\right.
$$

where $\tau=\xi_{0}, \sigma=\xi_{1}$. Our strategy will be to calculate the second variation of $F[\boldsymbol{c}(s)]$ and to derive its short-distance expansion as $s \rightarrow s^{\prime}$. After that we will translate the result to $A[\boldsymbol{c}(s)]$.

The equations of motion for (2.1) have the form

$$
\left\{\begin{array}{l}
\partial_{a}\left(\frac{1}{y^{2}} \partial_{a} \boldsymbol{x}\right)=0 \\
\partial^{2} y=\frac{1}{y}\left[\left(\partial_{a} y\right)^{2}-\left(\partial_{a} \boldsymbol{x}\right)^{2}\right] .
\end{array}\right.
$$

The boundary conditions are

$$
\left\{\begin{array}{l}
\boldsymbol{x}(0, \sigma)=\boldsymbol{c}(\sigma), \\
y(0, \sigma)=0 .
\end{array}\right.
$$

It is straightforward to check that the small $\tau$ expansion of the solution has the form (see 《1])

$$
\left\{\begin{array}{l}
\boldsymbol{x}(\tau, \sigma)=\boldsymbol{c}(\sigma)+\frac{1}{2} \boldsymbol{f}(\sigma) \tau^{2}+\frac{1}{3} \boldsymbol{g}(\sigma) \tau^{3}+\ldots \\
y(\tau, \sigma)=a(\sigma) \tau+\frac{1}{3} b(\sigma) \tau^{3}+\ldots
\end{array}\right.
$$


Direct substitution of (2.7) into (2.6) gives the relations

$$
\left\{\begin{array}{l}
a^{2}(\sigma)=\left(\boldsymbol{c}^{\prime}(\sigma)\right)^{2} \\
\boldsymbol{f}=\left(\boldsymbol{c}^{\prime}\right)^{2} \frac{d}{d \sigma}\left(\frac{\boldsymbol{c}^{\prime}}{\left(\boldsymbol{c}^{\prime}\right)^{2}}\right),
\end{array}\right.
$$

while the functions $b$ and $\boldsymbol{g}$ remain arbitrary. They must be determined from the global considerations. After this is done, all higher terms involved in the expansion (2.7) are uniquely determined. For example the term $y \sim(1 / 4) e(\sigma) \tau^{4}$ will be given by

$$
e(\sigma)=-\frac{2}{a}(\boldsymbol{f} \boldsymbol{g})-\frac{2}{3 a}\left(\boldsymbol{c}^{\prime} \boldsymbol{g}^{\prime}\right),
$$

etc.

The stress tensors (2.5) at the boundary are also easily determined. Substituting (2.7) into (2.5) we obtain

$$
\left\{\begin{array}{l}
\left.T_{\perp \perp}\right|_{\tau \rightarrow 0}=\frac{1}{a^{2}}\left[\boldsymbol{f}^{2}-\left(\boldsymbol{c}^{\prime} \boldsymbol{f}^{\prime}\right)-\left(a^{\prime}\right)^{2}+2 a b\right] \\
\left.T_{\perp \|}\right|_{\tau \rightarrow 0}=\frac{1}{a^{2}}\left(\boldsymbol{c}^{\prime} \boldsymbol{g}\right)
\end{array}\right.
$$

Notice that the terms $\sim 1 / \tau^{2}$ in $T$ cancel automatically due to (2.8). This is because $T$ is an analytic function which cannot have such a singularity.

One more relation which we need is the expression for the variational derivative $\delta F / \delta \boldsymbol{c}(s)$. From (2.1) by the standard integration by parts we obtain

$$
\begin{aligned}
\delta F & =-\int \frac{d \sigma}{y^{2}}\left[\partial_{\tau} x^{m} \delta x^{m}+\partial_{\tau} y \delta y\right]_{\tau \rightarrow 0} \\
& =-\int \frac{d \sigma}{a^{2} \tau^{2}}\left[\left(f_{m} \tau+g_{m} \tau^{2}\right) \delta x_{m}+\left(a+b \tau^{2}\right) \delta y\right]_{\tau \rightarrow 0} \\
& =-\frac{1}{\tau} \int\left[\frac{f_{m}}{a^{2}} \delta x_{m}+\frac{\delta a}{a}\right]-\int \frac{d \sigma}{a^{2}} g_{m} \delta x_{m}
\end{aligned}
$$

(we assume here that the contour remains at $y=0$ ). The first divergent term is zero due to (2.8), which means that the divergent part of this action is constant. We obtain

$$
\frac{\delta F}{\delta \boldsymbol{c}(\sigma)}=-\frac{\boldsymbol{g}(\sigma)}{a^{2}} .
$$




\section{Second variation of the Dirichlet functional}

In order to act with the loop operator, we must find the second variation of $F$. After substituting in (2.1) the expression

$$
x_{M} \Rightarrow x_{M}(\xi)+y(\xi) \psi_{M}(\xi)
$$

and expanding to the second order, we obtain after more or less standard calculations

$$
\begin{aligned}
& S=S_{0}+S_{1}^{b d r y}+S_{2}+S_{2}^{b d r y}, \\
& S_{2}=\frac{1}{2} \int d^{2} \xi\left[\left(\nabla_{\alpha} \psi_{M}\right)^{2}+r_{M N}(\xi) \psi_{M} \psi_{N}\right],
\end{aligned}
$$

where

$$
\begin{aligned}
\nabla_{\alpha} \psi_{M} & =\partial_{\alpha} \psi_{M}+\left(\omega_{\alpha}\right)_{M N} \psi_{N} \\
r_{M N} & =\frac{1}{y^{2}}\left[\left(\partial_{\alpha} x_{K}\right)^{2} \delta_{M N}-\partial_{\alpha} x_{M} \partial_{\alpha} x_{N}\right], \\
\left(\omega_{\alpha}\right)_{M N} & =\frac{1}{y}\left[\partial_{\alpha} x_{N} \delta_{M 0}-\partial_{\alpha} x_{M} \delta_{N 0}\right] .
\end{aligned}
$$

The terms $S_{1,2}^{b d r y}$ containing the first and second order boundary contributions are also easily calculated, but we do not need them below.

The second variation of the action $S$ with respect to the $\boldsymbol{c}(\sigma)$ will be obtained if we find the classical solution of the linearized problem with the action $S_{2}$

$$
-\nabla^{2} \psi_{M}+r_{M N} \psi_{N}=0
$$

with the boundary condition

$$
\left\{\begin{array}{l}
\left.\psi_{m}\right|_{\tau \rightarrow 0} \rightarrow \frac{\delta c_{m}(\sigma)}{y}=\frac{\delta c_{m}(\sigma)}{a(\sigma) \tau} \\
\left.\psi_{0}\right|_{\tau \rightarrow 0}=O(1)
\end{array}\right.
$$

When this is done, we will expand $\psi_{m}(\tau, \sigma)$ up to the term $\propto \tau^{2}$, which will give us the variation of the $\boldsymbol{g}$-factor and, according to (2.12), the second variation of $F$.

Another, equivalent strategy (which requires knowing $S_{2}^{b d r y}$ ) would be to substitute this solution in (3.2) and find the kernel of the resulting quadratic functional. The standard integration 
by parts gives in this case

$$
\begin{aligned}
\delta^{(2)} S & =\lim _{\tau \rightarrow 0}\left[-\frac{1}{2} \int \psi_{M}(\tau, \sigma) \nabla_{\tau} \psi_{M}(\tau, \sigma) d \sigma+S_{2}^{b d r y}\right] \\
& =\frac{1}{2} \int \varkappa_{m n}\left(\sigma, \sigma^{\prime}\right) \delta c_{m}(\sigma) \delta c_{n}\left(\sigma^{\prime}\right) d \sigma d \sigma^{\prime} .
\end{aligned}
$$

Here the kernel $\varkappa\left(\sigma, \sigma^{\prime}\right)$ can be expressed through the Green function of the equation (3.4).

This Green function is not known for a general classical solution $\boldsymbol{x}=\boldsymbol{x}(\xi), y=y(\xi)$. Fortunately, all we need for our task is its short distance expansion as $\sigma \rightarrow \sigma^{\prime}$. And this is relatively easy to obtain. Let us begin with the leading singularity. Substitution of (2.7) into (3.3) gives in the highest order

$$
\begin{aligned}
& \left(\omega_{1}\right)_{0 m} \approx \frac{c_{m}^{\prime}}{a \tau}, \\
& r_{m n} \approx \frac{1}{\tau^{2}}\left[2 \delta_{m n}-\frac{c_{m}^{\prime} c_{n}^{\prime}}{a^{2}}\right], \quad r_{00} \approx \frac{1}{\tau^{2}} .
\end{aligned}
$$

Substituting this into (3.2), we obtain in this approximation

$$
S_{2}=\frac{1}{2} \int d^{2} \xi\left[\left(\partial_{\tau} \psi_{M}\right)^{2}+\left(\partial_{\sigma} \psi_{M}\right)^{2}+\frac{2}{\tau^{2}}\left(\psi_{M}\right)^{2}+\frac{c_{m}^{\prime}}{\tau a}\left(\psi_{m} \partial_{\sigma} \psi_{0}-\psi_{0} \partial_{\sigma} \psi_{m}\right)\right] .
$$

We kept the terms $\sim 1 / \tau^{2}$ in the Lagrangian. As we will see in a moment, this is sufficient in the leading order. In this order, we can also neglect the $\sigma$-dependence of $c_{m}^{\prime}$. Then the action splits into two parts

$$
\begin{aligned}
& S_{2}=S^{\perp}+S^{\|}, \\
& S^{\perp}=\frac{1}{2} \int d^{2} \xi\left[\left(\partial_{\alpha} \psi_{i}\right)^{2}+\frac{2}{\tau^{2}}\left(\psi_{i}\right)^{2}\right], \\
& S^{\|}=\frac{1}{2} \int d^{2} \xi\left[\left(\partial_{\alpha} \psi_{0}\right)^{2}+\left(\partial_{\alpha} \psi_{1}\right)^{2}+\frac{2}{\tau^{2}}\left(\psi_{0}^{2}+\psi_{1}^{2}\right)+\frac{1}{\tau}\left(\psi_{1} \partial_{\sigma} \psi_{0}-\psi_{0} \partial_{\sigma} \psi_{1}\right)\right],
\end{aligned}
$$

where $i=2, \ldots, D$, and we chose the contour to run in the $x_{1}$ direction.

Let us now solve the Dirichlet problem for the action (3.9). In the case of $S^{\perp}$ we get

$$
\begin{aligned}
& \left(\partial_{\tau}^{2}-p^{2}\right) \psi_{i}-\frac{2}{\tau^{2}} \psi_{i}=0, \\
& \psi_{i}(p, \tau)=\tau^{-1}(1+|p| \tau) e^{-|p| \tau} \delta c_{i}(p),
\end{aligned}
$$

where we introduced the Fourier transformed quantities $\psi_{i}(p, \tau)$ instead of $\psi_{i}(\sigma, \tau)$. 
The longitudinal case is slightly more complex, but complex variables solve it. If we introduce $\Psi=\psi_{1}+i \psi_{0}$, we get

$$
\left(\partial_{\tau}^{2}-p^{2}\right) \Psi-\frac{2}{\tau^{2}} \Psi=\frac{2}{\tau} p \Psi
$$

The solution of this equation takes the form

$$
\begin{aligned}
& \Psi(p, \tau)=\tau^{-1} e^{-|p| \tau}\left[1+\theta(-p)\left(2|p| \tau+2 p^{2} \tau^{2}\right)\right] \delta c_{1}(p), \\
& \left\{\begin{array}{l}
\psi_{1}(p, \tau)=\frac{1}{2}\left[\Psi(p, \tau)+\Psi^{*}(-p, \tau)\right] \\
\psi_{0}(p, \tau)=\frac{1}{2 i}\left[\Psi(p, \tau)-\Psi^{*}(-p, \tau)\right] .
\end{array}\right.
\end{aligned}
$$

As we discussed above, all we need from these solutions are the $\boldsymbol{g}$-factors, that is the coefficients in front of $\tau^{2}$ as $\tau \rightarrow 0$.

Expanding expressions $(3.10)$ and (3.12), we get

$$
\left\{\begin{array}{l}
g_{i}(p)=|p|^{3} \delta c_{i}(p) \\
g_{1}(p)=-2|p|^{3} \delta c_{1}(p)
\end{array}\right.
$$

The functional (3.6) can be written in a convenient form if we introduce the Fourier transform

$$
\varkappa\left(\sigma, \sigma^{\prime}\right)=\int \tilde{\varkappa}\left(\frac{\sigma+\sigma^{\prime}}{2}, p\right) e^{i p\left(\sigma-\sigma^{\prime}\right)} d p .
$$

From (3.13) and (2.12) it follows that

$$
\tilde{\varkappa}_{m n}(p, \sigma) \underset{p \rightarrow \infty}{=} \frac{|p|^{3}}{\boldsymbol{c}^{\prime}(\sigma)^{2}}\left[2 \frac{c_{m}^{\prime} c_{n}^{\prime}}{\left(\boldsymbol{c}^{\prime}\right)^{2}}-\left(\delta_{m n}-\frac{c_{m}^{\prime} c_{n}^{\prime}}{\left(\boldsymbol{c}^{\prime}\right)^{2}}\right)\right],
$$

where we restored the general form of $c_{m}$.

Let us now discuss the range of validity of this formula. In its derivation we neglected many terms in (3.4). There are terms containing higher powers of $\tau$ coming from higher order terms in (2.7) (we kept only the first ones). Also, we neglected the $\sigma$-dependence of $c_{m}^{\prime}(\sigma)$ in (3.8).

This is legitimate, since the values of $\tau$ involved in our calculations are $\tau \lesssim 1 /|p|$. Hence, if we treat the $\tau$-correction as a perturbation in (3.8), we will obtain a contribution to $\varkappa(p, \sigma)$ suppressed by $1 /|p|$. The same is true for the $\sigma$-correction of $c_{m}^{\prime}(\sigma)$-if we expand $c_{m}^{\prime}(\sigma)=$ $c_{m}^{\prime}\left(\sigma_{0}\right)+c_{m}^{\prime \prime}(\sigma)\left(\sigma-\sigma_{0}\right)$, then in perturbation theory $\sigma-\sigma_{0} \Rightarrow i \partial / \partial p$ in the $(p, \sigma)$-representation. So, our conclusion is that (3.15) is indeed the leading singularity, and we need $|p| \gg\left|\boldsymbol{c}^{\prime \prime}\right| /\left|\boldsymbol{c}^{\prime}\right|$ for it to be valid. 


\section{Dimensional analysis}

In the previous section, we found the leading singularity $\sim|p|^{3}$ in $\widetilde{\varkappa}_{m n}(p, \sigma)$. However, this is just the beginning of the story, since to calculate the action of the loop operator we need to pick up terms $\sim p^{0}$ in $\widetilde{\varkappa}_{m m}(p, \sigma)$. The general structure has the form

$$
\widetilde{\varkappa}_{m m}(p, \sigma) \underset{p \rightarrow \infty}{=}(3-D) \frac{|p|^{3}}{\left(\boldsymbol{c}^{\prime}(\sigma)\right)^{2}}+A_{1}(\sigma) p^{2}+A_{2}(\sigma)|p|+A_{3}(\sigma)+\ldots
$$

According to our analysis, in order to calculate $A_{1}$ we have to expand $r_{M N}$ up to $1 / \tau$ and $\omega_{\alpha}$ up to $\tau^{0}$. $A_{2}$ and $A_{3}$ will require further expansion. We will also have to expand $\boldsymbol{c}^{\prime}(\sigma)$ to the needed order.

Direct use of the perturbation theory is straightforward because we know explicit Green functions for the unperturbed equations (3.10) and (3.11), but cumbersome (see Appendix).

We can greatly simplify our task by noticing that the functions $A_{1,2,3}(\sigma)$ depend locally on the properties of the coefficients $r_{M N}$ and $\omega_{\alpha}$ in (3.4) in the limit $\tau \rightarrow 0$. That means that they locally depend on the quantities $\boldsymbol{c}, \boldsymbol{f}, \boldsymbol{g}, a, b, \ldots$ appearing in the expansion (2.7). Moreover, we can uncover this dependence by the simple dimensional analysis.

The rules are as follows. We already know that

$$
\tau \sim \sigma \sim 1 / p
$$

where by $\sigma$ we mean deviation from the middle point. Then

$$
\begin{aligned}
& \boldsymbol{c}^{\prime}(\sigma) \sim \tau^{0}, \quad \boldsymbol{c}^{\prime \prime}(\sigma) \sim 1 / \tau, \quad \boldsymbol{c}^{\prime \prime \prime}(\sigma) \sim 1 / \tau^{2}, \\
& \boldsymbol{f} \sim 1 / \tau, \quad \boldsymbol{g} \sim 1 / \tau^{2}, \quad a \sim \tau^{0}, \quad b \sim 1 / \tau^{2} .
\end{aligned}
$$

Notice that with these assignments it follows from (2.10) that $T_{\perp \perp} \sim T_{\perp \|} \sim 1 / \tau^{2}$, as it should be.

Now, from (4.1) the scaling of $A_{1,2,3}$ must be the following:

$$
A_{1} \sim p \sim 1 / \tau, \quad A_{2} \sim p^{2} \sim 1 / \tau^{2}, \quad A_{3} \sim 1 / \tau^{3}
$$

Let us start now constructing these quantities. For $A_{1}$ the only thinkable combination is

$$
A_{1}=f\left(\boldsymbol{c}^{2}\right)\left(\boldsymbol{c}^{\prime} \boldsymbol{c}^{\prime \prime}\right)
$$


It follows that we must have $A_{1}=0$, because the expression (4.5) is odd under the change $\sigma \rightarrow-\sigma$, while the equations (3.4) and (3.6) preserve this parity. Hence we confirm the result of [1] that there is no term $\propto \delta^{\prime \prime}\left(\sigma_{1}-\sigma_{2}\right)$ in $\delta^{2} A / \delta \boldsymbol{c}\left(\sigma_{1}\right) \delta \boldsymbol{c}\left(\sigma_{2}\right)$ (this term would break the zigzag symmetry).

For $A_{2}$ we have

$$
A_{2}=\alpha_{1}\left(\boldsymbol{c}^{\prime \prime}\right)^{2}+\alpha_{2}\left(\boldsymbol{c}^{\prime} \boldsymbol{c}^{\prime \prime}\right)^{2}+\alpha_{3}\left(\boldsymbol{c}^{\prime} \boldsymbol{c}^{\prime \prime \prime}\right)+\alpha_{4} b+\alpha_{5}\left(\boldsymbol{c}^{\prime} \boldsymbol{g}\right) .
$$

Notice that $\alpha_{5}=0$ because $\boldsymbol{g}$ is even under $\sigma \rightarrow-\sigma$ while $\boldsymbol{c}^{\prime}$ is odd. If the Virasoro conditions are satisfied, we can express $b$ in terms of the contour from (2.10). The quantities $\alpha_{k}$ are functions of $\left(\boldsymbol{c}^{\prime}\right)^{2}$. It is easy to find them using the symmetry $\boldsymbol{c} \rightarrow \lambda \boldsymbol{c}$. Being a second derivative, $\varkappa$ must scale as $c^{-2}$. Hence we conclude that

$$
\alpha_{1} \propto\left(\boldsymbol{c}^{\prime}\right)^{-4}, \quad \alpha_{2} \propto\left(\boldsymbol{c}^{\prime}\right)^{-6}, \quad \alpha_{3} \propto\left(\boldsymbol{c}^{\prime}\right)^{-4}, \quad \alpha_{4} \propto\left|\boldsymbol{c}^{\prime}\right|^{-3} .
$$

To calculate the numerical coefficients, one may use the wavy line limit of [1]. We will not attempt it here.

Now we come to the most interesting term $A_{3}$, representing the action of the loop operator. We have the following possible structures:

$$
A_{3}=\beta_{1} \frac{\left(\boldsymbol{c}^{\prime \prime} \boldsymbol{g}\right)}{\left(\boldsymbol{c}^{\prime}\right)^{4}}+\beta_{2} \frac{\left(\boldsymbol{c}^{\prime} \boldsymbol{c}^{\prime \prime}\right)\left(\boldsymbol{c}^{\prime} \boldsymbol{g}\right)}{\left(\boldsymbol{c}^{\prime}\right)^{6}}+\beta_{3} \frac{\left(\boldsymbol{c}^{\prime} \boldsymbol{g}\right)^{\prime}}{\left(\boldsymbol{c}^{\prime}\right)^{4}} .
$$

Once again dimensionally possible terms $\left(\boldsymbol{c}^{\prime} \boldsymbol{c}^{(4)}\right),\left(\boldsymbol{c}^{\prime \prime} \boldsymbol{c}^{\prime \prime \prime}\right),\left(\boldsymbol{c}^{\prime \prime}\right)^{2}\left(\boldsymbol{c}^{\prime} \boldsymbol{c}^{\prime \prime}\right),\left(\boldsymbol{c}^{\prime} \boldsymbol{c}^{\prime \prime \prime}\right)\left(\boldsymbol{c}^{\prime} \boldsymbol{c}^{\prime \prime}\right), b^{\prime}$ and $b\left(\boldsymbol{c}^{\prime} \boldsymbol{c}^{\prime \prime}\right)$ are forbidden by parity. The main novelty here is the appearance of $\boldsymbol{g}$. This coefficient depends on $\boldsymbol{c}(\sigma)$ non-locally. However, we can express it from (2.12) as

$$
\boldsymbol{g}=-\left(\boldsymbol{c}^{\prime}\right)^{2}\left(\frac{\delta F}{\delta \boldsymbol{c}(\sigma)}\right) .
$$

Thus (4.8) gives a remarkable relation between the loop operator and the first derivative of $F$ :

$$
\widehat{L}(\sigma) F=-\frac{\beta_{1}}{\left(\boldsymbol{c}^{\prime}\right)^{2}}\left(\boldsymbol{c}^{\prime \prime} \frac{\delta F}{\delta \boldsymbol{c}}\right)+\ldots
$$

Since the remaining terms contain the combination

$$
\left(\boldsymbol{c}^{\prime} \boldsymbol{g}\right)=\left(\boldsymbol{c}^{\prime}\right)^{2} T_{\perp \|},
$$

they drop out if the Virasoro conditions are satisfied. 


\section{Second variation of the minimal area}

In order to calculate the minimal area, we have to use the relation (2.4). By taking $\alpha(s) \Rightarrow$ $s+\alpha(s)$ and $c_{m}(s) \Rightarrow c_{m}(s)+\delta c_{m}(s)$, and by expanding (2.4) to the second order, we obtain

$$
\begin{aligned}
\delta^{(2)} A= & \min _{\{\alpha(s)\}}\left[\int \frac{\delta F}{\delta c_{m}(s)}\left(\delta c_{m}^{\prime}(s) \alpha(s)+\frac{1}{2} c_{m}^{\prime \prime}(s) \alpha^{2}(s)\right) d s\right. \\
& \left.+\frac{1}{2} \int \frac{\delta^{2} F}{\delta c_{m}(s) \delta c_{n}\left(s^{\prime}\right)}\left(\delta c_{m}(s)+\alpha(s) c_{m}^{\prime}(s)\right)\left(\delta c_{n}\left(s^{\prime}\right)+\alpha\left(s^{\prime}\right) c_{n}^{\prime}\left(s^{\prime}\right)\right) d s d s^{\prime}\right] .
\end{aligned}
$$

In principle this formula solves the problem of expressing the second derivative of the minimal area $A$ through the Dirichlet functional $F$. For that we have to exclude $\alpha(s)$ from it by the use of extremality condition. Again this can be done by the use of the short distance expansions of the kernels involved. However, for our limited purposes in this paper we do not have to do it, since the dimensional analysis for $\widehat{L} A$ is the same as for $\widehat{L} F$.

We will discuss in Appendix direct calculation of $\widehat{L} A$, while here we can just conclude from (4.8) that

$$
\widehat{L} A[\boldsymbol{c}(s)]=\gamma \frac{\left(\boldsymbol{c}^{\prime \prime} \boldsymbol{g}\right)}{\left(\boldsymbol{c}^{\prime}\right)^{4}}=-\frac{\gamma}{\left(\boldsymbol{c}^{\prime}\right)^{2}}\left(\boldsymbol{c}^{\prime \prime}(s) \frac{\delta A[\boldsymbol{c}(s)]}{\delta \boldsymbol{c}(s)}\right)
$$

To calculate the constant $\gamma$, it is sufficient to consider the wavy line limit of [四]. In this case we already obtained

$$
\gamma=D-4
$$

We can also understand why $\gamma \propto D-4$ without any calculations. Namely, at $D=4$ the $\widehat{L}$ operator is conformally invariant, which means that not only $A[\boldsymbol{c}(s)]$ is invariant under conformal transformations but also $\widehat{L} A[\boldsymbol{c}(s)]$. [ H Hower these transformations can be used to set $\boldsymbol{c}^{\prime \prime}(\sigma)=0$ (a familiar example - we can make straight lines out of circles). Thus the only way in which (5.2) may be consistent with conformal symmetry is $\gamma=0$ at $D=4$. As it was shown in [1], if $D \neq 4$ there is an inhomogeneous term in the conformal transformation of $\widehat{L} A$ proportional to $(D-4)$. That explains the origin of (5.3).

\footnotetext{
${ }^{2}$ This property of $\widehat{L}$ is to be expected, since the Yang-Mills equations are conformally invariant exactly at $D=4$. For the direct (and not completely trivial) proof see [1].
} 
So, the formulae (5.2) and (5.3) solve the main problem addressed in this paper-verification of the loop equation in the WKB limit. This is a first step in deriving the loop equation for the full string theory functional integral. This problem requires much more concrete expressions both for the Wilson loop and for the string Lagrangians. We hope to address it in the future. Here we will just point out a general mechanism by which the non-linear terms in (1.2) can be generated. The equation $(4.10)$ tells us that $\widehat{L}(\sigma) F \propto T_{\perp \|}(\sigma)$. In the classical theory $T_{\perp \|}=0$. However in quantum theory expectation values $\left\langle T_{\perp \|}\right\rangle$ are dominated by the pinched disc 《4]. This must be the source of the non-linearity in the loop equations. However there are still some technical difficulties in implementing this idea.

\section{Acknowledgements}

The work of A.P. was partially supported by NSF grant PHY-98-02484.

\section{Appendix: Calculation of $\widehat{L} A$}

In this appendix we give a direct calculation of $\widehat{L} A$ along the lines of Section 3 . This will provide an independent check of (5.3), and also to some extent justify and exemplify the dimensional analysis method of Section 4 .

It will be convenient to switch from the conformal coordinates $(\sigma, \tau)$ to the static gauge

$$
x_{0}(t, s)=t
$$

The minimal surface is then given by

$$
\boldsymbol{x}(t, s)=\boldsymbol{c}(s)+\frac{1}{2} \boldsymbol{f}(s) t^{2}+\frac{1}{3} \boldsymbol{g}(s) t^{3}+\frac{1}{4} \boldsymbol{h}(s) t^{4}+\ldots
$$

A derivation similar to (2.11) shows that in this gauge

$$
\frac{\delta A}{\delta \boldsymbol{g}(s)}=\sqrt{\boldsymbol{c}^{\prime 2}} \boldsymbol{g}(s)
$$

To simplify further calculations, we choose the coordinate $s$ on the unperturbed world sheet so 
that

$$
\begin{cases}\boldsymbol{c}^{\prime 2}(s) \equiv 1 & \left.\Rightarrow \boldsymbol{f}=\boldsymbol{c}^{\prime \prime}\right) \\ \left(\dot{\boldsymbol{x}}(t, s) \boldsymbol{c}^{\prime}(s)\right) \equiv 0 & \left(\Rightarrow\left(\boldsymbol{f} \boldsymbol{c}^{\prime}\right)=\left(\boldsymbol{g} \boldsymbol{c}^{\prime}\right)=\left(\boldsymbol{h} \boldsymbol{c}^{\prime}\right)=\cdots=0\right) .\end{cases}
$$

Let us choose a tangent space basis $\left\{\boldsymbol{t}, \boldsymbol{n}^{a}\right\}, a=1, \ldots, D$, at every point of the curve $\boldsymbol{c}(s)$ so that

$$
\boldsymbol{t}=\frac{\boldsymbol{c}^{\prime}}{\sqrt{\boldsymbol{c}^{\prime 2}}}, \quad\left(\boldsymbol{n}^{a} \boldsymbol{t}\right)=0, \quad\left(\boldsymbol{n}^{a} \boldsymbol{n}^{b}\right)=\delta^{a b} .
$$

Then every variation $\delta \boldsymbol{c}$ can be decomposed into the normal and longitudinal part:

$$
\delta \boldsymbol{c}=\boldsymbol{n}^{a}\left(\boldsymbol{n}^{a} \delta \boldsymbol{c}\right)+\boldsymbol{t}(\boldsymbol{t} \delta \boldsymbol{c})=\delta \boldsymbol{c}^{\perp}+\delta \boldsymbol{c}^{\|} .
$$

The second variation of $A$ takes the form

$$
\begin{aligned}
\delta^{(2)} A= & \frac{1}{2} \iint \frac{\delta^{2} A}{\delta c_{i}(s) \delta c_{k}\left(s^{\prime}\right)} \delta c_{i}(s) \delta c_{k}\left(s^{\prime}\right) \\
= & \frac{1}{2} \iint \frac{\delta^{2} A}{\delta \boldsymbol{n}^{a}(s) \delta \boldsymbol{n}^{b}\left(s^{\prime}\right)}\left(\boldsymbol{n}^{a} \delta \boldsymbol{c}(s)\right)\left(\boldsymbol{n}^{b} \delta \boldsymbol{c}\left(s^{\prime}\right)\right) \\
& +2 \frac{\delta^{2} A}{\delta \boldsymbol{t}(s) \delta \boldsymbol{n}^{a}\left(s^{\prime}\right)}(\boldsymbol{t} \delta \boldsymbol{c}(s))\left(\boldsymbol{n}^{a} \delta \boldsymbol{c}\left(s^{\prime}\right)\right)+\frac{\delta^{2} A}{\delta \boldsymbol{t}(s) \delta \boldsymbol{t}\left(s^{\prime}\right)}(\boldsymbol{t} \delta \boldsymbol{c}(s))\left(\boldsymbol{t} \delta \boldsymbol{c}\left(s^{\prime}\right)\right),
\end{aligned}
$$

where

$$
\begin{aligned}
\frac{\delta^{2} A}{\delta \boldsymbol{n}^{a}(s) \delta \boldsymbol{n}^{b}\left(s^{\prime}\right)} & =\frac{\delta^{2} A}{\delta c_{i}(s) \delta c_{k}\left(s^{\prime}\right)} n_{i}^{a}(s) n_{k}^{b}\left(s^{\prime}\right), \\
\frac{\delta^{2} A}{\delta \boldsymbol{t}(s) \delta \boldsymbol{n}^{a}\left(s^{\prime}\right)} & =\frac{\delta^{2} A}{\delta c_{i}(s) \delta c_{k}\left(s^{\prime}\right)} t_{i}(s) n_{k}^{a}\left(s^{\prime}\right), \\
\frac{\delta^{2} A}{\delta \boldsymbol{t}(s) \delta \boldsymbol{t}\left(s^{\prime}\right)} & =\frac{\delta^{2} A}{\delta c_{i}(s) \delta c_{k}\left(s^{\prime}\right)} t_{i}(s) t_{k}\left(s^{\prime}\right) .
\end{aligned}
$$

Differentiating the reparametrization invariance condition $t_{i}(s) \cdot \delta A / \delta c_{i}(s)=0$ gives

$$
\frac{\delta^{2} A}{\delta c_{i}(s) \delta c_{k}\left(s^{\prime}\right)} t_{i}(s)=-\frac{\delta A}{\delta c_{i}(s)} \cdot \frac{\delta t_{i}(s)}{\delta c_{k}\left(s^{\prime}\right)} .
$$

Now it follows from (A.7)-(A.9) that

$$
\begin{aligned}
\frac{\delta^{2} A}{\delta c_{i}(s) \delta c_{i}\left(s^{\prime}\right)}= & \frac{\delta^{2} A}{\delta \boldsymbol{n}^{a}(s) \delta \boldsymbol{n}^{b}\left(s^{\prime}\right)}\left(\boldsymbol{n}^{a}(s) \boldsymbol{n}^{b}\left(s^{\prime}\right)\right) \\
& -\frac{\delta A}{\delta c_{i}(s)} \cdot \frac{\delta t_{i}(s)}{\delta c_{k}\left(s^{\prime}\right)}\left[2 n_{k}^{a}\left(s^{\prime}\right)\left(\boldsymbol{t}(s) \boldsymbol{n}^{a}\left(s^{\prime}\right)\right)+t_{k}\left(s^{\prime}\right)\left(\boldsymbol{t}(s) \boldsymbol{t}\left(s^{\prime}\right)\right)\right] \\
= & \frac{\delta^{2} A}{\delta \boldsymbol{n}^{a}(s) \delta \boldsymbol{n}^{b}\left(s^{\prime}\right)}\left(\boldsymbol{n}^{a}(s) \boldsymbol{n}^{b}\left(s^{\prime}\right)\right)+\left(\frac{\delta A}{\delta \boldsymbol{c}(s)} \boldsymbol{c}^{\prime \prime}(s)\right) \delta\left(s-s^{\prime}\right),
\end{aligned}
$$


where in the last line we used

$$
\frac{\delta t_{i}(s)}{\delta c_{k}\left(s^{\prime}\right)}=\left[\delta_{i k}-c_{i}^{\prime}(s) c_{j}^{\prime}(s)\right] \delta^{\prime}\left(s-s^{\prime}\right) .
$$

To find $\delta^{2} A / \delta \boldsymbol{n}^{a}(s) \delta \boldsymbol{n}^{b}\left(s^{\prime}\right)$, we vary (A.3) for the second time with a normal variation $\delta \boldsymbol{c}=\delta \boldsymbol{c}^{\perp}$. The result is

$$
\delta_{\perp}^{(2)} A=\frac{1}{2} \int\left[\left(\boldsymbol{g} \delta \boldsymbol{c}^{\perp}\right)\left(\boldsymbol{c}^{\prime \prime} \delta \boldsymbol{c}^{\perp}\right)-\left(\delta \boldsymbol{g} \delta \boldsymbol{c}^{\perp}\right)\right] d s
$$

From this we see that

$$
\frac{\delta^{2} A}{\delta \boldsymbol{n}^{a}(s) \delta \boldsymbol{n}^{a}\left(s^{\prime}\right)}=-\frac{\delta g^{a}(s)}{\delta \boldsymbol{n}^{a}\left(s^{\prime}\right)}+\left(\boldsymbol{g} \boldsymbol{c}^{\prime \prime}\right) \delta\left(s-s^{\prime}\right), \quad \delta g^{a}:=\left(\boldsymbol{n}^{a} \delta \boldsymbol{g}\right) .
$$

It follows from this and (A.10) that

$$
\widehat{L}(s) A=\text { coefficient before } \delta\left(s-s^{\prime}\right) \text { in }\left(-\frac{\delta g^{a}(s)}{\delta \boldsymbol{n}^{a}\left(s^{\prime}\right)}\right) .
$$

Thus we reduced the problem to studying how $\boldsymbol{g}(s)$ changes under normal variations.

It is well known (a clear-cut derivation can be found in $\mid 5$ ) that under a normal variation $x_{M} \Rightarrow x_{M}(t, s)+\psi_{M}(t, s)$ the area of a minimal surface in curved space changes to the second order in $\psi$ by

$$
S_{2}=\int\left|\nabla^{\perp} \psi\right|^{2}-\sum_{i, j}\left|\left\langle\psi, B\left(E_{i}, E_{j}\right)\right\rangle\right|^{2}-\left\langle R\left(E_{i}, \psi\right) \psi, E_{i}\right\rangle
$$

(modulo boundary terms). In this coordinate-free notation $\nabla^{\perp}$ is the covariant derivative in the normal bundle; $\left\{E_{i}\right\}$ is an orthonormal basis of the surface tangent space; $\langle\cdot, \cdot\rangle$ is the metric of the ambient space; $B\left(E_{i}, E_{j}\right)$ is the second fundamental form of the surface; $R$ is the Riemann curvature tensor; integration is taken with respect to the induced metric volume form.

Let us specify (A.15) to our case of the surface (A.1), A.2) in the AdS space. We assume that at every point $(t, s)$ of the surface we have a basis $\left\{n^{a}(t, s)\right\}$ of $(D-1)$ normal vectors such that

$$
n^{a}(0, s)=\left(\begin{array}{c}
0 \\
\boldsymbol{n}^{a}(s)
\end{array}\right), \quad n_{M}^{a} n_{M}^{b}=\delta^{a b},
$$

with $\boldsymbol{n}^{a}$ from above. We also put $\nu_{M}^{a}(t, s)=t n_{M}^{a}(t, s)$, so that $\nu^{a}$ are AdS-normalized. Now $\psi$ can be written as

$$
\psi_{M}=\psi^{a}(t, s) \nu_{M}^{a}(t, s)
$$


The curvature term in (A.15) is simple:

$$
\begin{aligned}
& R_{[M K][N L]}=-\left(G_{M N} G_{K L}-G_{M L} G_{K N}\right) \\
& \left\langle R\left(E_{i}, \psi\right) \psi, E_{i}\right\rangle=-\langle\psi, \psi\rangle\left\langle E_{i}, E_{i}\right\rangle+\left\langle\psi, E_{i}\right\rangle\left\langle\psi, E_{i}\right\rangle=-2 \psi^{a} \psi^{a} .
\end{aligned}
$$

For the kinetic term we have

$$
\begin{aligned}
& \int\left|\nabla^{\perp} \psi\right|^{2}=\int d^{2} \xi \sqrt{g} g^{\alpha \beta} \nabla_{\alpha} \psi^{a} \nabla_{\beta} \psi^{a} \\
& \nabla_{\alpha} \psi^{a}=\partial_{\alpha} \psi^{a}+\left(w_{\alpha}\right)^{[a b]} \psi^{b} .
\end{aligned}
$$

Here $g_{\alpha \beta}=\left\langle\partial_{\alpha} x, \partial_{\beta} x\right\rangle$ is the induced metric, which is found to be

$$
\begin{aligned}
g_{\alpha \beta} & =\frac{1}{t^{2}}\left(\begin{array}{cc}
1+\boldsymbol{f}^{2} t^{2}+2(\boldsymbol{f} \boldsymbol{g}) t^{3} & * \\
\frac{1}{2}\left(\boldsymbol{f} \boldsymbol{f}^{\prime}\right) t^{3} & 1-\boldsymbol{f}^{2} t^{2}-\frac{2}{3}(\boldsymbol{f} \boldsymbol{g}) t^{3}
\end{array}\right)+O\left(t^{2}\right) \\
\sqrt{g} & =\frac{1}{t^{2}}\left(1+\frac{2}{3}(\boldsymbol{f} \boldsymbol{g}) t^{3}\right)+O\left(t^{2}\right) .
\end{aligned}
$$

The spin connection coefficients $w_{\alpha}$ are antisymmetric and given by

$$
\left(w_{\alpha}\right)^{[a b]}=\left\langle\nabla_{\alpha} \nu^{a}, \nu^{b}\right\rangle=\partial_{\alpha} n_{M}^{a} \cdot n_{M}^{b} \quad(a \neq b)
$$

To simplify them, we choose the normals so that at the boundary

$$
\left(\boldsymbol{n}^{a}\right)^{\prime}=-\left(\boldsymbol{n}^{a} \boldsymbol{c}^{\prime \prime}\right) \boldsymbol{c}^{\prime}
$$

(it is easy to see that this condition is compatible with A.5)). For this choice of gauge we see from (A.21) that $w_{\alpha}=0$ at the boundary, and thus for small $t$

$$
w_{\alpha}(t, s)=O(t)
$$

Finally, the second term in (A.15) is $O\left(\psi^{2} K^{2}\right)$, where

$$
K^{2}(t, s)=\left\langle B\left(E_{i}, E_{j}\right), B\left(E_{i}, E_{j}\right)\right\rangle
$$

is the extrinsic curvature of the surface squared. This quantity is connected with the ambient space and the surface curvature tensors by the Gauss equation:

$$
K^{2}=\left\langle R\left(E_{i}, E_{j}\right) E_{j}, E_{i}\right\rangle-\left\langle r\left(E_{i}, E_{j}\right) E_{j}, E_{i}\right\rangle=-2-r
$$


where $r$ is the scalar curvature of the induced metric $g_{\alpha \beta}$. One can calculate from (A.20) that $r=-2+O\left(t^{4}\right)$, and thus

$$
K^{2}=O\left(t^{2}\right)
$$

By using (A.18), (A.19), (A.23) and (A.26) in (A.15) we obtain

$$
S_{2}=\int d^{2} \xi\left[\sqrt{g}\left(g^{\alpha \beta} \partial_{\alpha} \psi^{a} \partial_{\beta} \psi^{a}+2 g^{\alpha \beta} w_{\alpha}^{[a b]} \partial_{\beta} \psi^{a} \psi^{b}+2 \psi^{a} \psi^{a}\right)+O\left(t^{2} \psi^{2}\right)\right] .
$$

Varying (A.27), we obtain the equation of motion for $\psi$

$$
\partial_{\beta}\left(\sqrt{g} g^{\alpha \beta} \partial_{\alpha} \psi^{a}\right)-2 \sqrt{g} \psi^{a}+2 \sqrt{g} g^{\alpha \beta} w_{\alpha}^{[a b]} \partial_{\beta} \psi^{b}=O\left(t^{2} \psi\right)
$$

As it was explained in Section 3 (this will also be evident from the calculation below) each power of $t$ in the equations of motion suppresses the perturbative contribution to the Green function by a factor of $1 / p$. Since the highest singularity will be $|p|^{3}$ and we are interested in finding terms $\sim p^{0}$, the terms $O\left(t^{2} \psi\right)$ in (A.28) are negligible, being 4 orders less singular than the leading term $\sqrt{g} \psi^{a}$.

The third term in the LHS of (A.28) is in principle just 2 orders less singular than the leading terms, so its contribution to $\delta g^{a}(s) / \delta \boldsymbol{n}^{b}\left(s^{\prime}\right)$ can contain terms $\propto \delta^{\prime}\left(s-s^{\prime}\right)$ and $\delta\left(s-s^{\prime}\right)$. However, this contribution will be antisymmetric in $a \leftrightarrow b$ and hence is irrelevant for the subsequent substitution into (A.14).

Thus effectively we have to care only about the first two terms in (A.28). Taking this into account and using (A.20), we get the following equation for $\phi=\phi^{a}=t \cdot \psi^{a}$

$$
\begin{aligned}
\partial_{t} \frac{\dot{\phi}}{t^{2}}+\frac{\phi^{\prime \prime}}{t^{2}}= & \left(\boldsymbol{f}^{2}+\frac{4}{3}(\boldsymbol{f} \boldsymbol{g}) t\right)\left(\ddot{\phi}-\phi^{\prime \prime}\right) \\
& +\frac{4}{3}(\boldsymbol{f} \boldsymbol{g}) \dot{\phi}-\frac{3}{2}\left(\boldsymbol{f} \boldsymbol{f}^{\prime}\right) \phi^{\prime}+\left(\boldsymbol{f} \boldsymbol{f}^{\prime}\right) t \dot{\phi}^{\prime}+\text { negligible } .
\end{aligned}
$$

The boundary conditions are

$$
\phi(0, s)=\phi(s)
$$

The solution of the Dirichlet problem (A.29), (A.30) can be written as

$$
\phi(t, s)=\int K\left(t, s \mid s^{\prime}\right) \phi\left(s^{\prime}\right) d s^{\prime}
$$


where the Green function $K\left(t, s \mid s^{\prime}\right)$ solves (A.29) with the boundary conditions

$$
K\left(0, s \mid s^{\prime}\right)=\delta\left(s-s^{\prime}\right)
$$

The small $t$ expansion of $\phi$ can be found through $K$ as follows

$$
\phi(t, s)=\phi(s)+\int\left[t \dot{K}\left(0, s \mid s^{\prime}\right)+\frac{1}{2} t^{2} \ddot{K}\left(0, s \mid s^{\prime}\right)+\ldots\right] \phi\left(s^{\prime}\right) d s^{\prime} .
$$

In particular, we have

$$
\frac{\delta \dddot{\phi}^{a}(s)}{\delta \phi^{b}\left(s^{\prime}\right)}=\dddot{K}\left(0, s \mid s^{\prime}\right)+\text { antisymmetric in } a \leftrightarrow b
$$

To calculate $K$ perturbatively, we pass from the variables $\left(s, s^{\prime}\right)$ to $\left(\sigma=s-s^{\prime}, s^{\prime}\right)$ and perform the Fourier transform in $\sigma$. We have

$$
\partial_{s} \Rightarrow \partial_{\sigma} \Rightarrow i p, \quad s \Rightarrow \sigma+s^{\prime}=s^{\prime}+i \partial / \partial p .
$$

Now equation (A.29) takes the form

$$
\begin{aligned}
\partial_{t} \frac{\dot{K}}{t^{2}}-\frac{p^{2} K}{t^{2}}= & {\left[\boldsymbol{f}^{2}+2 i\left(\boldsymbol{f} \boldsymbol{f}^{\prime}\right) \partial_{p}+\frac{4}{3}(\boldsymbol{f} \boldsymbol{g}) t\right]\left(\ddot{K}+p^{2} K\right) } \\
& +\frac{4}{3}(\boldsymbol{f} \boldsymbol{g}) \dot{K}-\frac{3}{2}\left(\boldsymbol{f} \boldsymbol{f}^{\prime}\right) i p K+\left(\boldsymbol{f} \boldsymbol{f}^{\prime}\right) \text { tip } \dot{K}+\text { negligible }
\end{aligned}
$$

where the coefficients in the RHS are taken at $s^{\prime}$.

The unperturbed Green function is

$$
K_{0}=e^{-|p| t}(1+|p| t)
$$

We are looking for $K$ in the form of a series

$$
K\left(p, t \mid s^{\prime}\right)=K_{0}+K_{1}+K_{2}+K_{3}+\ldots,
$$

where

$$
K_{i} \sim \frac{1}{p^{i}} e^{-|p| t} \quad(p \gg 1) .
$$

The equation (A.36) can be schematically written as

$$
A_{0} K=A_{2} K+A_{3} K+\ldots
$$


where we indicated the relative order of singularity of the differential operators. It follows by simple counting that the $K_{1}$ correction is absent from (A.38) and that $\widetilde{K}=K_{2}+K_{3}$ is found from the equation

$$
\left\{\begin{array}{l}
A_{0} \widetilde{K}=\left(A_{2}+A_{3}\right) K_{0} \\
\left.\widetilde{K}\right|_{t=0}=0
\end{array}\right.
$$

Thus substituting $K_{0}$ into the RHS of (A.36) gives the equation for $\widetilde{K}$

$$
\begin{aligned}
\partial_{t} \frac{\partial_{t} \widetilde{K}}{t^{2}}-\frac{p^{2} \widetilde{K}}{t^{2}}=f(p, t):=2 \boldsymbol{f}^{2} p^{2} e^{-|p| t}|p| t \\
\quad+|p| e^{-|p| t}\left\{\frac{8}{3}(\boldsymbol{f} \boldsymbol{g}) p^{2} t^{2}-\frac{4}{3}(\boldsymbol{f} \boldsymbol{g})|p| t+i \operatorname{sign} p\left(\boldsymbol{f} \boldsymbol{f}^{\prime}\right)\left(\frac{9}{2}|p| t-3 p^{2} t^{2}-\frac{3}{2}\right)\right\} .
\end{aligned}
$$

This inhomogeneous problem has the solution

$$
\widetilde{K}(p, t)=\int G_{p}(t, \tau) f(p, \tau) d \tau
$$

with the Green function

$$
\left\{\begin{array}{l}
G_{p}\left(t_{1}, t_{2}\right)=\frac{1}{2|p|^{3}} F_{0}\left(|p| t_{<}\right) F_{\infty}\left(|p| t_{>}\right), \\
F_{\infty}(t)=e^{-t}(1+t) \\
F_{0}(t)=e^{t}(1-t)-F_{\infty}(t) .
\end{array}\right.
$$

Routine power expansion shows that

$$
\left(K_{0}+\widetilde{K}\right)(p, t)=1-\frac{1}{2} t^{2} p^{2}+\frac{1}{3} t^{3}\left(|p|^{3}-\boldsymbol{f}^{2}|p|-(\boldsymbol{f} \boldsymbol{g})-\left(\boldsymbol{f} \boldsymbol{f}^{\prime}\right) i \operatorname{sign} p\right) .
$$

Thus it follows from this and (A.34) that

$$
\frac{\delta \phi^{a}(s)}{\delta \phi^{b}\left(s^{\prime}\right)}=-2(\boldsymbol{f} \boldsymbol{g}) \delta\left(s-s^{\prime}\right)+\ldots
$$

Finally, it remains to relate $\delta \dddot{\phi}^{a} / \delta \phi^{b}$ and $\delta g^{a} / \delta \phi^{b}$. This relation is not immediate, because the normal variation does not preserve the static gauge (A.1). The perturbed surface

$$
\left\{\begin{array}{l}
\widetilde{x}_{0}(t, s)=t+\phi^{a}(t, s) n_{0}^{a}(t, s), \\
\widetilde{\boldsymbol{x}}(t, s)=\boldsymbol{x}(t, s)+\phi^{a}(t, s) \boldsymbol{n}^{a}(t, s)
\end{array}\right.
$$

has to be reparametrized by introducing the new coordinate $\widetilde{t}=\widetilde{x}_{0}$, so that

$$
t=\tilde{t}-\phi^{a}(\widetilde{t}, s) n_{0}^{a}(\widetilde{t}, s)+O\left(\phi^{2}\right) .
$$


In the new coordinates the perturbed surface takes the static gauge form (we rename $\widetilde{t} \rightarrow t$ )

$$
\left\{\begin{array}{l}
\widetilde{x}_{0}(t, s)=t \\
\widetilde{\boldsymbol{x}}(t, s)=\boldsymbol{x}(t, s)+\phi^{a}(t, s) \boldsymbol{n}^{a}(t, s)-\left(\boldsymbol{f} t+\boldsymbol{g} t^{2}\right) \phi^{a}(t, s) n_{0}^{a}(t, s) .
\end{array}\right.
$$

To use (A.49), we must explicitly find $n^{a}(t, s)$ satisfying (A.16). The result is non-unique if $D>2$. Our choice was to first transform $n^{a}(0, s) \Rightarrow \widetilde{n}^{a}(t, s)$ for each $a$ by adding a linear combination of $\dot{x}(t, s), x^{\prime}(t, s)$ so that $\widetilde{n}^{a}$ are orthogonal to the surface, and then to orthonormalize the family $\left\{\widetilde{n}^{a}\right\}$. The result is:

$$
\left\{\begin{aligned}
n_{0}^{a}(t, s)= & -f_{a} t-g_{a} t^{2}+O\left(t^{3}\right) \\
\boldsymbol{n}^{a}(t, s)= & \boldsymbol{n}^{a}+\frac{1}{2} \boldsymbol{n}^{b}\left(f_{a} f_{b} t^{2}+\left(f_{a} g_{b}+f_{b} g_{a}\right) t^{3}\right) \\
& -\boldsymbol{f} f_{a} t^{2}-\left(\boldsymbol{g} f_{a}+\boldsymbol{f} g_{a}\right) t^{3}+\boldsymbol{c}^{\prime} O\left(t^{2}\right)+O\left(t^{4}\right)
\end{aligned}\right.
$$

(all functions in the RHS are taken at $s$ ). Substituting this into (A.49) gives

$$
\delta g^{a}=\frac{1}{2} \dddot{\phi}^{a}+\frac{3}{2}\left(f_{a} g_{b}+f_{b} g_{a}\right) \phi^{b} .
$$

Now it follows from (A.46) and (A.51) that

$$
\frac{\delta g^{a}(s)}{\delta \phi^{a}\left(s^{\prime}\right)}=(4-D) \delta\left(s-s^{\prime}\right)+\ldots
$$

and hence $\gamma=D-4$ by (A.14).

\section{References}

[1] A. Polyakov, V. Rychkov, Nucl. Phys. B581 (2000), 116-134, hep-th/0002106

[2] J. Maldacena, Phys. Rev. Lett. 80 (1998) 4859, hep-th/9803002

[3] S.-J. Rey, J. Yee, hep-th/9803001

[4] A. Polyakov, hep-th/9711002 (Proceedings of "Strings 97")

[5] T.H. Colding, W.P. Minicozzi, Minimal surfaces, New York University, 1999.

[6] N. Drukker, D.J. Gross, H. Ooguri, Phys. Rev. D60 (1999) 125006, hep-th/9904191 